\title{
Iron line afterglows: How to produce them
}

\author{
D. Lazzati $^{1,2}$, S. Campana ${ }^{1}$, and G. Ghisellini ${ }^{1}$ \\ 1 Osservatorio Astronomico di Brera, Via Bianchi 46, I-23807 Merate (Lc), Italy \\ 2 Dipartimento di Fisica, Università degli Studi di Milano, Via Celoria 16, I-20133 Milano, Italy
}

Received December 29, 1998; accepted June 9, 1999

\begin{abstract}
We discuss how a powerful iron line emission can be produced if $\sim 1-5$ iron rich solar masses are concentrated in the close vicinity of the burst. Recombination, thermal and fluorescent reflection are discussed. We find that recombination suffers the high Compton temperature of the plasma while the other two scenarios are not mutually exclusive and could account for the claimed iron line detected in two afterglows.
\end{abstract}

Key words: gamma-ray: burst

\section{Introduction}

Piro et al. (1998) and Yoshida et al. (1998) (this volume) report the detection of an iron emission line in the $\mathrm{X}$-ray afterglow spectrum of two $\gamma$-ray bursts, GRB 970508 and GRB 970828. The detection of strong iron emission lines would unambiguously point towards the presence of a few per cent of iron solar masses concentrated in a compact region in the vicinity of the burster. The simplest way to account for this is to assume the presence of a very young supernova remnant. Thus the presence of such a line in the $\mathrm{X}$-ray afterglow spectrum would represent the "Rosetta Stone" for unveiling the burst progenitor. There are three main classes of models for the origin of GRB: Neutron Star-Neutron Star (NS-NS) mergers (Eichler et al. 1989), Hypernovae (Paczyński 1998) and Supranovae (Vietri \& Stella 1998).

$\mathrm{X}$-ray line emission following GRB events has been recently discussed in the Hypernova scenario by Ghisellini et al. (1998) and Boettcher et al. (1998). None of these works predict, with reasonable assumptions on the burst surroundings, iron lines strong enough to be detectable during the $\mathrm{X}$-ray afterglow. Moreover, the line emission should last over a time-scale of years given the size of the emitting nebula. On the contrary, the Supranova accounts

Correspondence to: lazzati@merate.mi.astro.it in a quite natural way for the presence of a massive remnant in the close vicinity of the bursting source.

Here we discuss three possibilities for the iron line origin: $i$ ) recombination of the supernova shell iron atoms, photoionized by the burst photons; ii) thermal emission of the shell, which, once heated by the burst emission, produces an iron blended line (mainly due to FeXXV and FeXXVI); iii) fluorescence of a very dense, compact and relatively cold supernova shell observed in reflection.

We assume that $\sim 1-5$ iron rich solar masses are concentrated in the burst surroundings. Some general constraints about the required mass and the size of the emitting region are discussed in Ghisellini et al. (1998) (this volume), whereas a more detailed description can be found in Lazzati et al. (1999). The cosmological parameters will be set throughout this letter to $H_{0}=65 \mathrm{~km} \mathrm{~s}^{-1} \mathrm{Mpc}^{-1}$, $q_{0}=0.5$ and $\Lambda=0$.

\section{Emission mechanisms}

\subsection{Recombination}

If the plasma can remain cold and dense enough and if the recombination and ionization times are comparable, a strong iron line can be obtained through recombination during the burst with a reasonable amount of iron. A recombination time of almost $10^{-2} \mathrm{~s}$ is needed to produce a line visible during the afterglow.

The recombination time of an hydrogenic ion of atomic number $Z$ in a thermal plasma is $t_{\mathrm{rec}}=\left(\alpha_{\mathrm{r}} n\right)^{-1}$ (Verner \& Ferland 1986), where $n$ is the electron density and $\alpha_{\mathrm{r}}$ is the temperature dependent recombination coefficient (e.g. Seaton 1959). For a relatively cold and dense iron plasma with $T=10^{4} \mathrm{~K}, t_{\mathrm{rec}} \sim 0.14 n_{10}^{-1} \mathrm{~s}$ while, for $T=10^{8} \mathrm{~K}$, $t_{\text {rec }} \sim 10^{2} n_{10}^{-1} \mathrm{~s}$. Hence, while a cold plasma could have the necessary recombination rate to ensure the needed fast photon production, this mechanism would be almost completely damped at higher temperatures with reasonable values for the electron density.

The main problem with this interpretation is that the temperature of the plasma absorbing a sizeable fraction 
of the burst energy is bound to be large, unless an extreme large density enhances the radiative cooling rates. In fact the Compton equilibrium temperature (assuming typical burst high energy spectra) is of the order of a few times $10^{8} \mathrm{~K}$. Note that the scattering optical depth $\tau_{\mathrm{T}}$ is bound to be in the range $0.1-1$ since a sizable fraction of the burst energy must be absorbed without an excessive smearing of the emission lines.

We conclude that the case of a strong iron line due to repeated photoionization and recombination events during the burst emission faces the problem of a temperature too large to ensure the required fast recombination rate.

\subsection{Thermal emission from the surrounding shell}

We assume for simplicity that the shell is homogeneous and compact and that it is heated up to $T=10^{8} T_{8} \mathrm{~K}$ by the burst photons. We must require that $\tau_{\mathrm{T}} \simeq 0.1 \div 1$, implying a shell radius $R \geq 810^{15}\left(M / M_{\odot}\right)^{1 / 2}$. In this conditions the shell would emit a broad band bremsstrahlung continuum with several emission lines overlaid (Raymond \& Smith 1977), the most relevant being the $6.7 \mathrm{keV}$ iron blend. Analogous spectra are observed in cluster of galaxies (Sarazin 1988), but the higher iron abundance expected in a supernova shell would enhance line emission.

The equivalent width (EW) of the line in a solar abundance plasma has been carefully computed by Bahcall \& Sarazin (1978; see in particular their Fig. 1) and ranges from several tens of $\mathrm{eV}$ at high $\left(510^{8} \mathrm{~K}\right)$ temperatures to $\sim 2 \mathrm{keV}$ at $2.510^{7} \mathrm{~K}$. A very weak line is expected for temperature lower than $510^{6} \mathrm{~K}$. For temperatures larger than $510^{7} \mathrm{~K}$ the EW dependence on temperature can be reasonably approximated as a power law. Assuming an iron abundance 10 times solar we have:

$\mathrm{EW}(T) \simeq 3.8 T_{8}^{-1.9} \mathrm{keV} \quad\left(T_{8} \geq 0.5\right)$.

Considering the bremsstrahlung intensity at $6.7 \mathrm{keV}$, we obtain a line luminosity of:

$L_{\mathrm{Fe}} \simeq 810^{42} \exp \left(-\frac{0.8}{T_{8}}\right)\left(\frac{M}{M_{\odot}}\right)^{2} V_{48}^{-1} T_{8}^{-2.4} \mathrm{erg} \mathrm{s}^{-1}$.

Therefore a shell of $M \sim 5 M_{\odot}$, typical for many type II SN (see Raymond 1984; Weiler \& Sramek 1988; Woosley 1988; McCray 1993), at a temperature slightly below $10^{8}$ $\mathrm{K}$ can produce a line flux of $10^{-13} \mathrm{erg} \mathrm{cm}^{-2} \mathrm{~s}^{-1}$ for $z=1$ bursts. The EW with respect to the underlying bremsstrahlung radiation would be a few $\mathrm{keV}$, but any other emission component (e.g. afterglow emission) would decrease the line EW. The line emission process can be stopped after about one day, if the afterglow photons enhance the plasma cooling via inverse Compton, lowering the temperature down to less than $10^{7} \mathrm{~K}$. Line emission can also be quenched by the reheating produced by the incoming fireball.

\subsection{Reflection}

In Seyfert galaxies we see a fluorescence $6.4 \mathrm{keV}$ iron line produced by a relatively cold $\left(T<10^{6} \mathrm{~K}\right)$ accretion disk, illuminated by a hot corona, which provides the ionizing photons (e.g. Ross \& Fabian 1993). In this case we need a scattering optical depth $\tau_{\mathrm{T}}>1$ of the fluorescent material and a size large enough to allow the line being emitted even $\sim$ one day after the GRB event (i.e. $R>10^{15} \mathrm{~cm}$ ).

In this model the emission line is produced only during the burst event, but in the observer frame it lasts for $R / c$. The observed luminosity of the Compton reflection component is equal to the $\sim 10 \%$ of the absorbed energy, divided by the time $R / c: L \sim 310^{45} E_{\text {abs, } 51} / R_{15} \mathrm{erg} \mathrm{s}^{-1}$. The luminosity in the iron line (see e.g. Matt et al. 1991) is $\sim 1 \%$ of this, times the iron abundance in solar units. Therefore the reflection component can contribute to the hard X-ray afterglow emission, and the iron line can have a luminosity up to $310^{44} \mathrm{erg} \mathrm{s}^{-1}$, corresponding to fluxes $\sim 10^{-13} \mathrm{erg} \mathrm{cm}^{-2} \mathrm{~s}^{-1}$ for a $z=1$ burst.

\section{Conclusions}

We discussed three possible mechanisms for the production of a strong iron line, visible during the $\mathrm{X}$-ray afterglows of GRB. All mechanisms require the presence of a large amount of iron in a compact region. The remnant of a supernova exploded a few months before the burst matches the required matter and size.

We find that the multiple ionization and recombination scenario has difficulties in reconciling the low temperature required to have a fast recombination with the large heating due to the burst flux. On the other hand, we believe that the other two alternatives (i.e. thermal emission and reflection) are promising and not mutually exclusive.

In the reflection scenario, afterglow spectra should show the typical hardening of the spectrum above a few $\mathrm{keV}$, and the line duration should be short.

\section{References}

Bahcall J.N., Sarazin C.L., 1978, ApJ 219, 781

Boettcher M., et al., 1998, A\&A (submitted), astro-ph/9809156

Eichler D., et al., 1989, Nat 340, 126

Ghisellini G., Haardt F., Campana S., Lazzati D., Covino S., 1998, ApJ (submitted), astro-ph/9808156

Lazzati D., Ghisellini G., Campana S., 1999, MNRAS 304, L31

Matt G., Perola G.C., Piro L., 1991, A\&A 247, 25

McCray R., 1993, ARA\&A 31, 175

Paczyński B., 1998, ApJ 494, L45

Raymond J.C., 1984, ARA\&A 22, 75

Raymond J.C., Smith B.W., 1977, ApJS 35, 419

Ross R., Fabian A.C., 1993, MNRAS 261, 74

Sarazin C.L., 1988, X-ray emission from clusters of galaxies. Cambridge: Cambridge University Press

Seaton M.J., 1959, MNRAS 119, 81

Verner D.A., Ferland G.J., 1996, ApJS 103, 467

Vietri M., Stella L., 1998, ApJ 507, L45

Weiler K.W., Sramek R.A., 1988, ARA\&A 26, 295

Woosley S.E., 1988, ApJ 330, 218 